\title{
An experimental testbed for the study of hydrodynamic issues in supernovae*
}

\author{
H. F. Robey, ${ }^{\dagger}{ }^{1}$ J. O. Kane, ${ }^{1}$ B. A. Remington, ${ }^{1}$ R. P. Drake, ${ }^{2}$ O. A. Hurricane,${ }^{1}$ H. Louis, ${ }^{1}$ \\ R. J. Wallace, ${ }^{1}$ J. Knauer ${ }^{3}$ P. Keiter, ${ }^{2}$ D. Arnett, ${ }^{4}$ and D. D. Ryutov ${ }^{1}$ \\ ${ }^{1}$ Lawrence Livermore National Laboratory, Livermore, California 94550 \\ ${ }^{2}$ University of Michigan, Ann Arbor, Michigan 48105 \\ ${ }^{3}$ Laboratory for Laser Energetics, University of Rochester, Rochester, New York 14623 \\ ${ }^{4}$ University of Arizona, Tucson, Arizona
}

(Received 24 October 2000; accepted 8 January 2001)

\begin{abstract}
More than a decade after the explosion of supernova 1987A, unresolved discrepancies still remain in attempts to numerically simulate the mixing processes initiated by the passage of a very strong shock through the layered structure of the progenitor star. Numerically computed velocities of the radioactive ${ }^{56} \mathrm{Ni}$ and ${ }^{56} \mathrm{Co}$, produced by shock-induced explosive burning within the silicon layer, for example, are still more than 50\% too low as compared with the measured velocities. To resolve such discrepancies between observation and simulation, an experimental testbed has been designed on the Omega Laser for the study of hydrodynamic issues of importance to supernovae (SNe). In this paper, results are presented from a series of scaled laboratory experiments designed to isolate and explore several issues in the hydrodynamics of supernova explosions. The results of the experiments are compared with numerical simulations and are generally found to be in reasonable agreement. (C) 2001 American Institute of Physics. [DOI: 10.1063/1.1352594]
\end{abstract}

\section{INTRODUCTION}

In February of 1987, a spectacular burst of light from supernova (SN) 1987A was first observed, subsequently generating an enormous wealth of observational data on this phenomenon. These observations strongly suggested that extensive mixing of the inner layers into the outer layers of the progenitor star had occurred. This material mixing was indicated by several forms of observation. X-ray emission from ${ }^{56} \mathrm{Ni}$ and ${ }^{56} \mathrm{Co}$, generated in the shock-induced explosive burning within the silicon layer, was observed at about six months, ${ }^{1-3}$ whereas one-dimensional spherically symmetrical numerical simulations predicted observation at approximately one year. ${ }^{4,5}$ The peak velocities of ${ }^{56} \mathrm{Co}$ were observed to be well in excess of $3000 \mathrm{~km} / \mathrm{s}^{6,7}$ Early twodimensional numerical simulations, by comparison, produced peak velocities of less than $2000 \mathrm{~km} / \mathrm{s}^{8-12}$ As initially shown by Chevalier, ${ }^{13}$ the dominant mechanisms responsible for the mixing in all of these simulations are the Rayleigh-Taylor (RT) $)^{14,15}$ and Richtmyer-Meshkov $(\mathrm{RM})^{16,17}$ instabilities. More recent and extensive twodimensional (2D) simulations, ${ }^{18}$ beginning just after core bounce, initially find very large velocities (up to $4000 \mathrm{~km} / \mathrm{s}$ ) of the newly created Fe group elements, but after impact with the reverse shock at the He-H interface, the velocities are again decelerated to a value below $2000 \mathrm{~km} / \mathrm{s}$. Thus the discrepancy between observation and simulation persists.

A number of possible explanations for these discrepancies are under active exploration. More detailed 2D simulations of the pre-existing convectively driven structure at the edge of the oxygen layer ${ }^{19-21}$ have been performed to better

*Paper CI1 2, Bull. Am. Phys. Soc. 45, 58 (2000).

${ }^{\dagger}$ Invited speaker. quantify the level of the initial perturbations. The mixing which results from shock propagation through this layer, however, was still found insufficient to explain the observations. For single-mode perturbations, three-dimensional effects are known from theoretical, numerical, and experimental work to increase the mixing over $2 \mathrm{D}$ perturbations. ${ }^{22-25}$ Initial 3D simulations of the SN mixing problem, ${ }^{12,26}$ however, still underpredict the material velocities.

Two possibilities exist for resolving this problem. The first possibility is that the assumptions underlying previous numerical simulations are incorrect or contain incomplete physics. An example is the recent work of Khokhlov et al. ${ }^{27}$ which brings the additional effects of rotation and magnetic fields into the problem. The resulting collapse launches a highly asymmetric explosion with bipolar jets and the formation of bow shocks and Mach disks and demonstrates that the assumption of spherical symmetry may be incorrect. The second possibility is that existing computer models may be incorrectly calculating the hydrodynamic evolution of the system. All numerical methods necessarily include approximations in the underlying equations. Different schemes use different orders of accuracy, employ nonphysical artificial viscosities to assure stability of the calculations, and, in spherical geometry, can suffer from vanishing metrics ${ }^{18,26}$ and grid-induced numerical instability. ${ }^{18,28}$ Numerical results often agree at the largest scales of motion, but can differ strongly at smaller scales (see, for example, Refs. 29, 30). In an effort to address this second possibility, laboratory experiments have been initiated ${ }^{31-35}$ to provide a set of benchmark data with which to validate the performance of astrophysical codes.

The purpose of the present paper is twofold. The first goal is to establish the range of phenomena relevant to the supernova mixing problem which may be addressed on a 


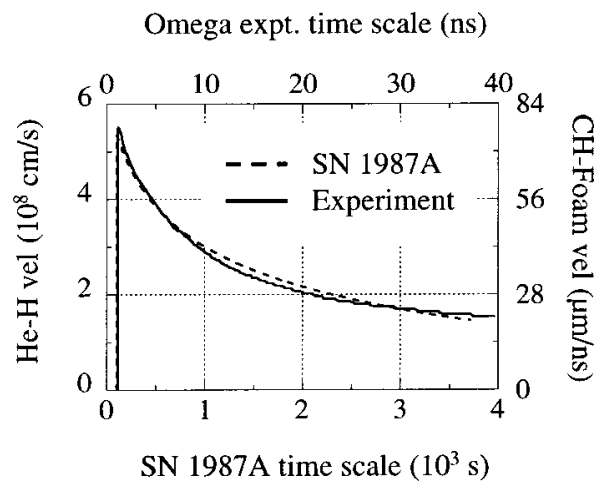

FIG. 1. Comparison of the SN He-H interface velocity (PROMETHEUS simulation) with the corresponding scaled laboratory experiment interface velocity (HYADES simulation).

laser facility. To that end, we present initial results from a series of scaled laboratory experiments designed to isolate and explore four separate issues of relevance to SN mixing. The first experiment explores the effects of spherical divergence on the instability evolution. The second studies the possibility of coupling between two spatially separated interfaces. The third compares and quantifies the difference between instability growth in two and three dimensions. Finally, the fourth begins to look at the evolution of an interface of more complicated modal content. The second goal of this work is to serve as a first rough assessment of the validity of numerical codes used for the simulation of supernova hydrodynamics. For each experiment, comparisons are made with numerical simulation, and the implications and relevance to the SN mixing problem are assessed.

\section{SCALING ISSUES}

Conner and Taylor, ${ }^{36}$ and more recently Ryutov et al. ${ }^{37,38}$ presented general scaling relations that govern the validity of laboratory scale experiments designed to replicate hydrodynamic phenomena occurring in astrophysical systems. The essential idea discussed in these papers is the following. In any system where dissipative processes due to viscosity, thermal conductivity, and radiative transport are small in comparison with the inertial transport terms, the equations of motion governing the evolution of the plasma reduce to the Euler equations. If, in addition, the plasma equation of state can be described as a polytropic gas with $p \sim \rho^{\gamma}$ (where $\gamma$ is the adiabatic index), then the Euler equations are scale invariant provided that the parameter $(L / t) /(P / \rho)^{1 / 2}$ remains constant. Here $L, t, P$, and $\rho$ are characteristic length, time, pressure, and density scales of the system, respectively. Any two systems for which this parameter has the same value and which have the same initial conditions will then evolve in an identical manner.

An example of such a scaling from the astrophysical to the laboratory scale is shown in Fig. 1. Figure 1 compares the numerically simulated interface velocity histories of the $\mathrm{SN} \mathrm{He}-\mathrm{H}$ interface computed using the astrophysical code PROMETHEUS ${ }^{9}$ with that of a scaled laboratory experiment computed using the one-dimensional radiationhydrodynamics code HYADES. ${ }^{39}$ Full details of the experi-

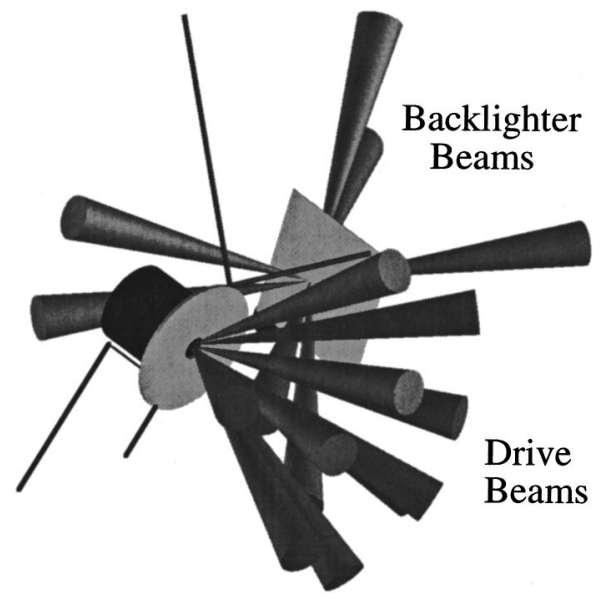

FIG. 2. 3D CAD drawing of the experimental configuration showing the target package and x-ray backlighter foil with the Omega beams used for initiating the strong shock conditions (drive) and diagnosing the experiment (backlighter beams).

ment will be given in Sec. IV. For the present discussion of scaling issues, we simply note the essential elements of the experimental setup. The more dense He layer in the SN progenitor is simulated in the experiment with a hemispherical shell of polystyrene $(\mathrm{CH})$ with a density $\rho=1.37 \mathrm{~g} / \mathrm{cm}^{3}$. The surrounding $\mathrm{H}$ layer is simulated with a low density $(\rho=0.1$ $\mathrm{g} / \mathrm{cm}^{3}$ ) carbon foam. In the Omega experiment, a strong shock is driven through the interface between these two materials by laser radiation. Passage of this shock through the interface initially accelerates the interface generating Richtmyer-Meshkov instability. The surrounding H (carbon foam) envelope then subsequently decelerates the interface. As is seen, the temporal evolution of both systems is quite similar. If the initial conditions of the interface (i.e., perturbation wavelengths and amplitudes) are also scaled, then the resulting evolution of any instabilities will also evolve in a similar manner.

\section{CONFIGURATION OF THE EXPERIMENTAL TESTBED}

The experiments are conducted on the Omega Laser at the Laboratory for Laser Energetics (LLE), University of Rochester. $^{40-43}$ Figure 2 shows an illustration of the generic experimental setup common to all four of the Omega experiments discussed in this report. The target package differs from experiment to experiment. The specific details of each target will be given in Sec. IV.

The strong shock conditions of interest are achieved by directing either six beams (divergent experiment) or ten beams (all other experiments) with a nominal measured energy of 420-500 J/beam (again experiment dependent) at a laser wavelength of $0.351 \mu \mathrm{m}$ on to the target. Each beam has a super-Gaussian spatial intensity profile defined as $I / I_{0}=\exp [-(r / 412 \mu \mathrm{m})]^{4.7}$. The combined spatial profile of all drive beams also follows this profile, with $I_{0}=(5-9)$ $\times 10^{14} \mathrm{~W} / \mathrm{cm}^{2}$. The intensity is reasonably constant over a central diameter of $600 \mu \mathrm{m}$, and falls off by about $10 \%$ by $800 \mu \mathrm{m}$. The typical diameter of the targets, by comparison, 
is $800 \mu \mathrm{m}$. Since considerable laser energy extends laterally beyond the diameter of the target packages, a shield (either $\mathrm{Au}$ or $\mathrm{Be}$ ) with an outer diameter of $2.5 \mathrm{~mm}$ and an inner aperture of $440 \mu \mathrm{m}$ (divergent) or $950 \mu \mathrm{m}$ (planar) was used to delay the propagation of a shock around the sides of the target. This proved to be successful in generating both spherical shock propagation through a hemispherical capsule as well as planar shock propagation for the planar targets. For all experiments, the temporal pulse is nominally flat with a duration of $1 \mathrm{~ns}$. The strength of the shock generated by this laser drive is quite strong, with initial pressures as determined from a one-dimensional HYADES simulation of approximately 50 Mbars.

The evolution of the instability at interfaces within the targets was diagnosed with $\mathrm{x}$-rays generated by directing an additional seven Omega beams onto a thin backlighter foil located $4 \mathrm{~mm}$ from the center of the target, as shown in Fig. 2. These beams, driven by a separate oscillator, were delayed in time relative to the drive beams by up to $100 \mathrm{~ns}$ to observe the instability evolution at late times. The contrast generated by differential absorption of the backlighter $\mathrm{x}$-rays by the target materials was imaged with a gated framing camera. ${ }^{44}$

\section{EXPERIMENTAL RESULTS}

\section{A. The effect of spherical divergence}

In order to investigate the effect of spherical divergence on the evolution of instability at an interface, we use targets consisting of a hemispherical shell of 3\% Ge-doped polystyrene $(\mathrm{CH})$ with a density $\rho=1.37 \mathrm{~g} / \mathrm{cm}^{3}$ surrounded by a volume of lower density foam. The capsule inner diameter (ID) is nominally $440 \mu \mathrm{m}$, and the outer diameter (OD) is $660 \mu \mathrm{m}$, giving a shell thickness of approximately $110 \mu \mathrm{m}$. The capsule is embedded within a cylinder of carbonized resorcinol formaldehyde (CRF), a porous foam with density $\rho=0.1 \mathrm{~g} / \mathrm{cm}^{3}$. The CRF cylinder measures $1500 \mu \mathrm{m}$ in diameter by $1500 \mu \mathrm{m}$ in length. An initial "two-dimensional" perturbation is imposed on the outer surface of the $\mathrm{CH}(\mathrm{Ge})$ capsule. The perturbation, produced by laser ablation, has a wavelength $\lambda=70 \mu \mathrm{m}$ and a peak-to-valley amplitude of $a_{P / V}=10 \mu \mathrm{m}$. The ripples form parallel grooves in the outer $\mathrm{CH}(\mathrm{Ge})$ surface with the crests aligned parallel with the diagnostic line of sight. Five cycles of the perturbation are included covering a projected square area of $350 \mu \mathrm{m} \times 350$ $\mu \mathrm{m}$ on the hemispherical surface. Figure 3(a) shows an illustration of the geometry of the perturbed hemisphere. This geometry provides a clear, well-defined side-on view of the ripple amplitude for back illuminated radiography. The disadvantage is that the perturbation is fully three-dimensional with two-dimensional ripples imposed on a spherical surface and is therefore difficult to simulate numerically.

Figure 3 shows experimental radiographs of the instability evolution obtained with a Sc backlighter foil generating $4.3 \mathrm{keV}$ He- $\alpha$ X-rays. In Fig. 3(b) an unperturbed capsule is shown at $t=13.6 \mathrm{~ns}$ as a reference case. The experiment magnification is fairly low $(4 \times)$ in order to obtain a large enough field of view to observe the shape of the entire expanding capsule. The capsule shell is shown to remain intact with no evidence of instability growth, indicating that the

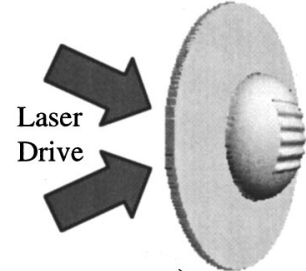

a.)

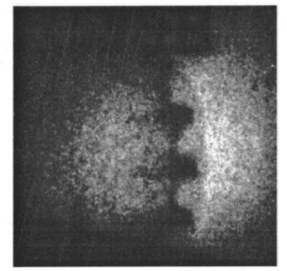

c.)

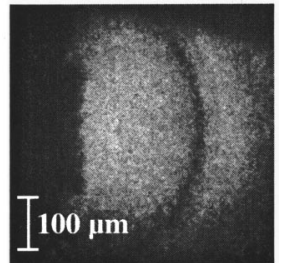

b.)

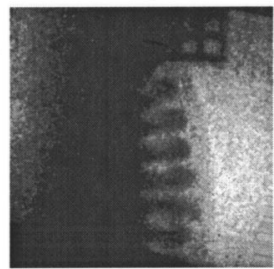

d.)
FIG. 3. (a) Schematic illustration of the divergent experiments; (b) experimental radiograph of an unperturbed capsule ( $4 \times$ magnification, $t=13 \mathrm{~ns}$ ); (c) radiograph of a perturbed capsule with $\lambda=70 \mu \mathrm{m}, a_{P-V}=10 \mu \mathrm{m}$ ( $8 \times$ magnification, $t=13 \mathrm{~ns}$ ); (d) radiograph of an equivalent planar target with same initial perturbation and drive as (c).

laser drive is sufficiently uniform. By comparison with the initial capsule position, the shell radius has expanded to $R / R_{0}=2.7$, and the capsule thickness has decreased to approximately half its initial value. The expanded capsule shape is seen to be very nearly spherical as well. The spherical shock is also seen in the image just outside the expanding shell.

Figure 3(c) shows the corresponding image at the same time, $t=13.6 \mathrm{~ns}$, for a capsule with a $\lambda=70 \mu \mathrm{m}$ and nominal $a_{P / V}=10 \mu \mathrm{m}$ perturbation. Again, the radial divergence factor is 2.7. The wavelength of the perturbation grows by an identical factor. The magnification for this image was increased to $8 \times$ to better reveal the details of the perturbation evolution. At this magnification, one can now clearly see the full extent of the perturbation and its proximity to the shock. The shape of the perturbed interface shows rather broad bubbles and spikes, more characteristic of a much lower Atwood number flow. The reason for this is attributed to the proximity of the shock, which effectively acts as a rigid boundary suppressing growth of the $\mathrm{CH}(\mathrm{Ge})$ spikes relative to the growth of the foam bubbles. This result can be compared with an equivalent planar interface, as shown in Fig. 3(d), where a $108 \mu \mathrm{m}$ thick planar layer of $4.3 \%$ Br-doped $\mathrm{CH}$ was accelerated with a nominally identical drive into the same $0.1 \mathrm{~g} / \mathrm{cm}^{3} \mathrm{CRF}$. Although the observation time is the same in Figs. 3(c) and 3(d), several distinct differences are observed between the spherically diverging and the planar cases. The wavelength remains much closer to the initially imposed wavelength in the planar case, although there is still some divergence due to shock diffraction effects. The shape of the spikes is also observed to be significantly more elongated with more pronounced "mushroom caps." The shock in this case is seen to be somewhat further from the interface as well.

Numerical simulations of this experiment were performed with a 2D Arbitrary Lagrangian-Eulerian radiationhydrodynamics code $\left(\mathrm{CALE}^{45}\right)$. Since an extensive discussion 


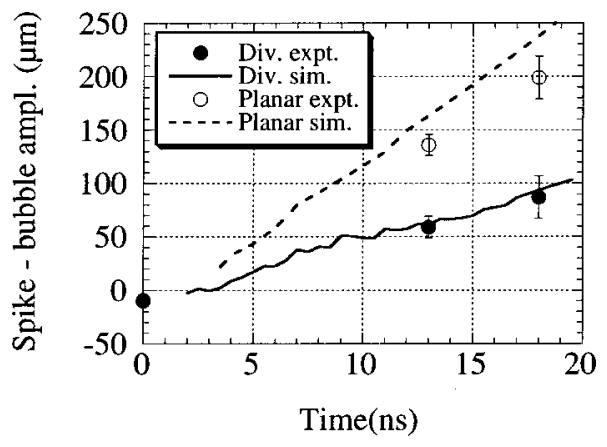

FIG. 4. Comparison of peak-to-valley amplitude of interface instability between spherically divergent and planar experiments.

of the numerical simulation of these experiments is given in Drake et $a l .{ }^{46}$ for the divergent experiments and Kane et al. ${ }^{47}$ for the planar experiments, we confine our discussion here to the results of the simulations. Figure 4 shows a plot of the amplitude of the perturbation for $t=13 \mathrm{~ns}$ and $18 \mathrm{~ns}$ for both the spherically diverging and the planar experiments. The result of the $2 \mathrm{D}$ numerical simulation is shown as well. The agreement of the numerical simulation and the experimental measurements is seen to be quite good for the divergent experiment. This is perhaps somewhat surprising since the $2 \mathrm{D}$ CALE simulations employ a cylindrically symmetrical perturbation (a "bulls-eye" pattern), as opposed to the truly 3D perturbation geometry of the experiment.

For the planar case, the simulation overpredicts the measured value by approximately $20 \%$. The most likely source of this discrepancy is in the experimental measurement of the perturbation amplitude. As seen in Fig. 3(b), the location of the radiographically opaque spike tips is very clearly resolved. The full extent of the transparent bubbles, however, is not well resolved. Since the diagnostic is looking through $1500 \mu \mathrm{m}$ of doped plastic, and there is a slight divergence of the interface, we cannot accurately measure the full extent of the bubbles. Future experiments will employ a radiographic tracer layer (to be described in the next section) which greatly improves this type of measurement. For the present, however, we note that in both the experiments and simulations, the instability growth is significantly reduced in spherically divergent geometry. This is primarily due to the increase in the perturbation wavelength as noted earlier, but also possibly by the proximity of the shock, which is observed to be much closer to the spike tips in the divergent experiment as compared to the planar case. Additional experiments are being planned to quantify this effect of shock proximity in reducing perturbation growth rates.

\section{B. Coupling between interfaces}

The second experiment considers the enhancement in mixing due to coupling between spatially separated interfaces. Coupling can occur either by the impact of structure from a previous interface, ${ }^{26}$ or as is investigated here, by the propagation of a perturbed shock originating from the passage through a previously perturbed interface.

Figure 5(a) shows an exploded view of the target used for the experiments. In order to approximate the decreasing

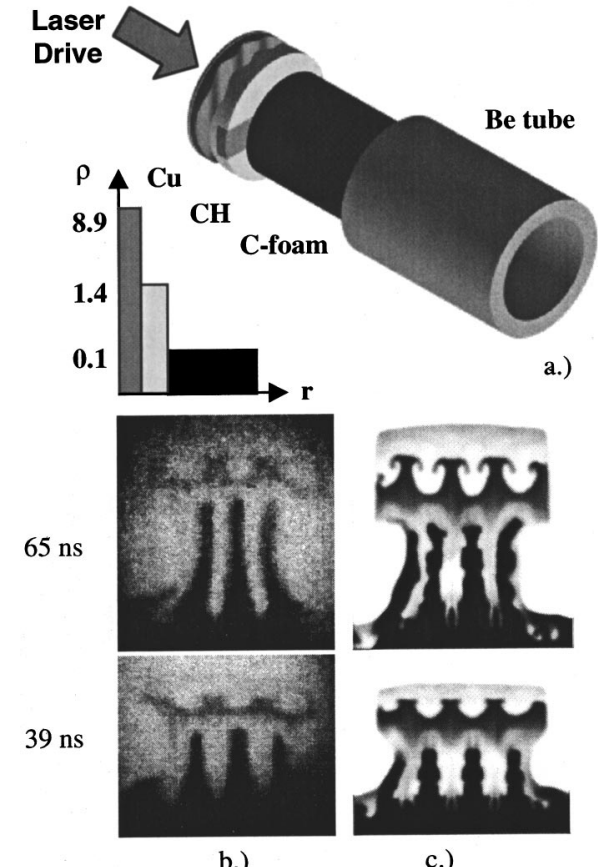

b.)

c.)

FIG. 5. (a) Schematic illustration of the interface coupling experiments; (b) experimental radiographs at $t=39$ and $65 \mathrm{~ns}$; (c) 2D PROMETHEUs simulated radiograph of the experiment.

radial density profile of a star, a target is prepared which consists of a series of layers of decreasing density mounted within a Be shock tube. The presence of the shock tube improves the planarity of the experiment by decreasing the lateral expansion of the target materials. The shock tube has an OD of $1100 \mu \mathrm{m}$ and an ID of $800 \mu \mathrm{m}$. Beginning at the laser drive end, the target consists of a $10 \mu \mathrm{m}$ polystyrene ablator layer $\left(\rho=1.05 \mathrm{~g} / \mathrm{cm}^{3}\right)$ followed by a $90 \mu \mathrm{m} \mathrm{Cu}$ layer $(\rho=8.9$ $\left.\mathrm{g} / \mathrm{cm}^{3}\right)$, a $150 \mu \mathrm{m}$ polyimide layer $\left(\rho=1.41 \mathrm{~g} / \mathrm{cm}^{3}\right)$, with the remainder of the target filled with a low density CRF foam $\left(\rho=0.1 \mathrm{~g} / \mathrm{cm}^{3}\right)$. Embedded within the polyimide layer is a radiographically opaque tracer strip of $4.3 \%$ brominated polystyrene. The tracer layer measures $75 \mu \mathrm{m}$ in the direction along the Be tube, and is $200 \mu \mathrm{m}$ wide along the diagnostic line-of-sight direction. The density of this $\mathrm{CH}(\mathrm{Br})$ layer $\left(\rho=1.42 \mathrm{~g} / \mathrm{cm}^{3}\right)$ is nearly identical to that of the surrounding polyimide. When viewed in side-illuminated radiography, nearly all of the contrast comes from this opaque tracer layer, allowing visualization of the shock-imprinted structure at interface 2 over only the central $200 \mu \mathrm{m}$ of the target. This helps to eliminate wall effects that are inherent in such an integrated line-of-sight diagnostic. ${ }^{48}$ A perturbation of wavelength $\lambda=200 \mu \mathrm{m}$ and amplitude $a_{P / V}=30 \mu \mathrm{m}$ is pre-imposed at the $\mathrm{Cu}$-polyimide interface. The perturbation at this interface grows due to Rayleigh-Taylor and Richtmyer-Meshkov instabilities, but the focus of this experiment is on the possibility of imprinting a perturbation at the second initially unperturbed interface.

The target is driven by ten beams of the Omega laser with a measured average energy of $420 \mathrm{~J} / \mathrm{beam}$ in a $1 \mathrm{~ns}$ pulse at a laser wavelength of $0.351 \mu \mathrm{m}$. For ten beams overlapped at target chamber center, the peak intensity is 
$8.9 \times 10^{14} \mathrm{~W} / \mathrm{cm}^{2}$. A $75 \mu \mathrm{m}$ thick, $2.5 \mathrm{~mm}$ diameter beryllium shield with a central aperture of $950 \mu \mathrm{m}$ diameter is mounted at the front of the target. The average intensity incident on the $\mathrm{CH}$ ablator over this central aperture is 5.7 $\times 10^{14} \mathrm{~W} / \mathrm{cm}^{2}$. The strength of the resulting shock by the time it impacts the $\mathrm{Cu}-\mathrm{CH}$ interface as determined from a one-dimensional HYADES simulation is approximately 10 Mbars.

The evolution of both the instability at the $\mathrm{Cu}$ /polyimide interface and the shock-imprinted perturbation at the $\mathrm{CH}(\mathrm{Br}) / \mathrm{CRF}$ interface were diagnosed with $6.7 \mathrm{keV}$ x-rays from a $12 \mu \mathrm{m}$ thick Fe backlighter foil located $4 \mathrm{~mm}$ from the center of the target. The diagnostic beams were delayed relative to the drive beams by up to $91 \mathrm{~ns}$ to observe the instability evolution at late times. Figure 5(b) shows the experimental radiographs obtained at $t=39$ and $65 \mathrm{~ns}$ after the drive beams. The shock propagation direction is upward in the figure. Growth of the perturbation at the $\mathrm{Cu}-\mathrm{CH}$ interface is clearly seen as the three black spikes $(\mathrm{Cu})$ interpenetrating the bubbles of polyimide at the bottom of each image. A partial fourth $\mathrm{Cu}$ spike is also visible at the left of the image. This spike is highly distorted due to the presence of large vortices at the wall of the tube. Such wall vortices, generated by the interaction of reflected waves and boundary layers at the shock tube walls, are also observed in conventional gasdynamic shock tubes as is discussed in Brouillette and Bonnaza. ${ }^{48}$ The dark region located above the $\mathrm{Cu}$ spikes is the brominated $\mathrm{CH}$ tracer layer. An imprinted perturbation of opposite phase is clearly seen at the interface between this layer and the shocked foam. The separation between the two interfaces was deliberately designed to generate this phase reversal of the imprinted perturbation in order to distinguish it from any possible imprinting that might arise from the growth of structure at the first interface. At $t=65 \mathrm{~ns}$, the instability at both interfaces has continued to evolve, much of the growth at the first interface $(\mathrm{Cu}-\mathrm{CH})$ being due to the rarefaction wave reflected from the second interface.

The results from 2D numerical simulations using the astrophysical code PROMETHEUS are shown at the same times as the experimental images. The agreement is seen to be excellent. The magnitude, phase, and shape of the imprinted structure at the second interface are all very similar at both times. There is a small difference in the fourth distorted $\mathrm{Cu}$ spike as the wall vortex in the experimental image is somewhat larger than in the simulation. Small differences can also be observed concerning secondary structure on both the $\mathrm{Cu}$ spikes and the roll-up of $\mathrm{CH}(\mathrm{Br})$ at the second interface at 65 ns. The resolution of the data is insufficient to provide a comparison with the simulations concerning such fine structure. As with the previous experiment, we conclude that the code does a very good job of simulating the phenomenon of shock imprinting.

While this experiment is useful for benchmarking code performance, it differs in one important regard from the SN problem. The possibility of imprinting by a rippled shock depends very strongly on the density profile through which the shock propagates. In our experiment, the shock propagated through constant density material before impacting the second interface. As is well known, planar shock propagation through constant density materials with an adiabatic index $\gamma>1.2$ is stable. ${ }^{49,50}$ (For $\gamma<1.2$, however, instability has been shown by theory, ${ }^{51}$ numerical simulation, ${ }^{52}$ and experiment ${ }^{53}$ to be possible in self-similar adiabatic blast wave profiles.) In the supernova, the value of the adiabatic index in the vicinity of the He-H interface falls in the range $4 / 3<\gamma<5 / 3$ as shown by Müller et al., ${ }^{10}$ and thin-shell blast wave instabilities will not play a role. The decreasing radial density profile in the star, by contrast, is significant and can cause portions of the shock located radially further from the center to propagate faster than regions located closer to the center. Thus shocks can be unstable in a decreasing density profile. This problem has been analyzed by Chevalier ${ }^{54,55}$ for an exponentially decaying density profile and by Sari et al. ${ }^{56}$ for power law profiles $\rho \sim r^{-\omega}$, where $\omega>3$. In both cases, shock instability was shown to be possible for perturbations whose wavelength is much longer than the scale height, overstable for more moderate values of the wavelength, and stable for small wavelengths.

For shock imprinting to occur, we do not require instability of the shock, but merely a small enough decay rate for some perturbation to survive to the second interface. An exact comparison of the possibility of shock coupling in SN 1987A, for example, would require a stability analysis of the spatially varying density profile of that particular progenitor star. From the general form of the stability analyses of Chevalier et al. and Sari et al., however, it can be concluded that shock imprinting will most likely occur for the longest wavelength perturbations. This leads one to consider, as mentioned in Sec. I, the possibility of nonsymmetrical explosion scenarios with very low mode-number azimuthal perturbations. The jet mechanism of Khokhlov ${ }^{27}$ is an extreme case of such a low mode-number perturbation.

\section{The effect of dimensionality (2D vs 3D)}

We turn now to the role of dimensionality in the instability evolution. As mentioned earlier, nearly all simulations to date have been performed in 2D, the only exceptions being those in Refs. 12 and 26. It is well known from simple buoyancy-drag models ${ }^{23}$ that single-mode perturbation growth in three dimensions is greater than in two. The additional mixing arising in a three-dimensional flow could be a potential candidate to explain the SN mixing problem. In order to quantify the contribution of dimensionality, experiments with two equivalent targets have been conducted. Both targets consist of a $108 \mu \mathrm{m}$ thick layer of $4.3 \%$ brominated polystyrene followed by a $1500 \mu \mathrm{m}$ long cylinder of CRF. The densities of the two materials are the same as used previously. The only difference between the two targets is in the perturbation imposed at the interface, as shown in Figs. 6(a), 6(b). The 2D target contains a single sinusoidal perturbation of wavelength $50 \mu \mathrm{m}$ and peak-to-valley amplitude of $5 \mu \mathrm{m}$. The 3D target contains a perturbation with two orthogonal sinusoids, both of wavelength $71 \mu \mathrm{m}$ and overall amplitude $5 \mu \mathrm{m}$. The perturbations were chosen such that the wave number magnitude is the same for each giving identical RM and RT growth in the linear regime. 


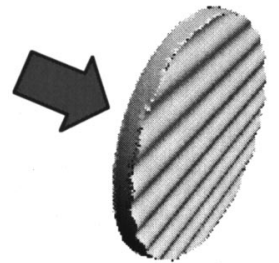

a.)

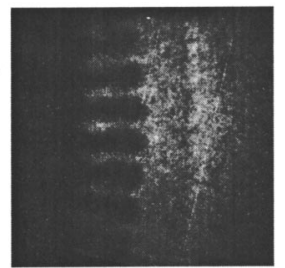

c.)

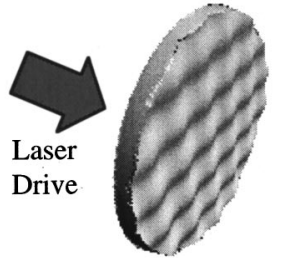

b.)

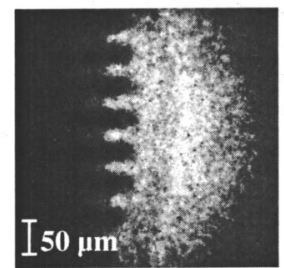

d.)
FIG. 6. Schematic illustration of (a) 2D and (b) 3D interface perturbation; (c) experimental radiograph of 2D interface at $13 \mathrm{~ns}$; (d) experimental radiograph of $3 \mathrm{D}$ interface at $13 \mathrm{~ns}$.

Experimental radiographs obtained using $4.7 \mathrm{keV}$ x-rays from a Ti backlighter foil are shown in Figs. 6(c) for the 2D target and 6(d) for the 3D target. For both of these targets, no radiographic tracer layer was used, so the images give the integrated differential x-ray absorption through $1500 \mu \mathrm{m}$ of $\mathrm{CH}(\mathrm{Br})$. The target alignment is obviously quite good as the images are looking along $2 \mathrm{D}$ ridges 30 times longer than the imposed wavelength of $50 \mu \mathrm{m}$. The lack of a radiographic tracer tends to obscure the full extent of the bubbles of the lower density foam, however. From these images, then, we are only able to obtain information concerning the extent of the spikes of denser material.

A measure of the difference in growth is obtained from Figs. 6(c), 6(d) by comparing the distance between the spike tips and the shock. The relevant dimensions from each experiment are given in Table I. The average measured drive energy per beam is within $5 \%$ for the two shots. Using either the theoretically predicted scaling ${ }^{57}$ for the ablation pressure $\left(P \sim I^{2 / 3}\right)$ or the experimentally measured value ${ }^{58,59}(P$ $\sim I^{0.8}$, combined with the fact that the velocity scales as the square root of the pressure, the difference in interface velocities should be less than $2 \%$. From the measured position of the reference grids, the shock locations in each case are within $10 \mu \mathrm{m}$, which is of order of the experimental error. The distance of the spike tips relative to the shock reference is $84 \mu \mathrm{m}$ for the $2 \mathrm{D}$ case and $70 \mu \mathrm{m}$ for the $3 \mathrm{D}$ case, indicating that the 3D spikes have grown approximately $20 \%$ more.

TABLE I. Experiment parameters for the 2D vs 3D comparison.

\begin{tabular}{lll}
\hline \multicolumn{1}{c}{ Parameter } & 2D & $3 \mathrm{D}$ \\
\hline Drive energy/beam $(\mathrm{J})$ & 534.2 & 505.9 \\
$\mathrm{CH}(\mathrm{Br})$ layer thickness $(\mu \mathrm{m})$ & 156 & 161 \\
Downstream grid position $(\mu \mathrm{m})$ & 791 & 732 \\
Shock position $(\mu \mathrm{m})$ & 757 & 767 \\
Spike position $(\mu \mathrm{m})$ & 673 & 697 \\
Shock-to-spike distance $(\mu \mathrm{m})$ & 84 & 70 \\
\hline \hline
\end{tabular}

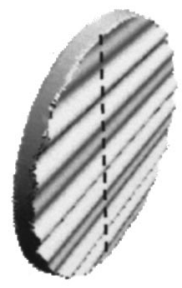

a.)

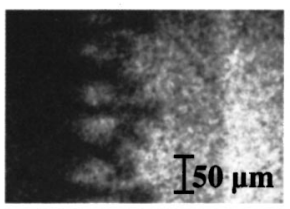

c.)

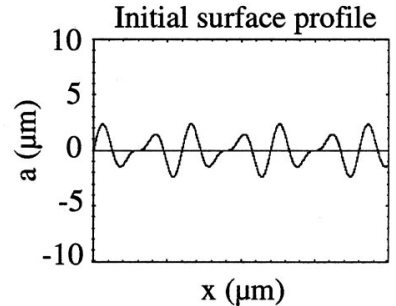

b.)

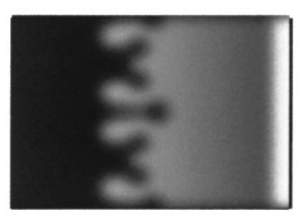

d.)
FIG. 7. (a) Schematic illustration of the two-mode interface perturbation; (b) 1D lineout through the interface; (c) experimental radiograph of the interface at $13 \mathrm{~ns}$; (d) 2D CALE simulation of the interface at $13 \mathrm{~ns}$.

A numerical simulation of this particular experiment has not yet been performed. We can compare with previous numerical studies of similar geometries, however. In Kane et $a l .,{ }^{26}$ for example, the growth of perturbations at the $\mathrm{He}-\mathrm{H}$ interface of SN 1987A was studied using the PROMETHEUS code. Their numerical results also show an increase in the spike growth in 3D over that in 2D, although their increase was approximately $30 \%$ as compared with the $20 \%$ observed in the present experiments. Their conclusion with regard to the SN mixing problem, however, was that even a $30 \%$ increase in the extent of the mixing region was insufficient to explain the observations of SN 1987A. The same conclusion pertains to the present experimental results as well.

\section{The effect of modal content (two-mode experiment)}

A final experiment was conducted to begin to explore the evolution of a more complicated interfacial structure. Thus far, we have only investigated single-mode interface perturbations. In the SN mixing problem, however, the interface is almost certain to be fully turbulent. A first step toward such an interface structure is to introduce two modes which, through nonlinear mode coupling, will then begin to generate additional modes in the spectrum, eventually producing a fully turbulent flow.

The configuration for this experiment is very similar to that just described for the previous $2 \mathrm{D}$ experiment with the radiographic tracer layer. The only difference was in the interface perturbation. Two sinusoidal modes with wavelengths of $\lambda_{1}=60 \mu \mathrm{m}$ and $\lambda_{2}=40 \mu \mathrm{m}$ with initial amplitudes of 1.5 and $1.0 \mu \mathrm{m}$, respectively, were machined onto the interface. Figure 7(a) shows an illustration of the machined surface. A lineout through the surface is given in Fig. 7(b). An experimental radiograph obtained using $4.7 \mathrm{keV}$ $\mathrm{X}$-rays from a Ti backlighter is shown in Fig. 7(c) for $t$ $=13 \mathrm{~ns}$. The simulated radiograph from the corresponding CALE simulation, at the same time, is shown in Fig. 7(d). The interface structure is considerably more complicated than for the single-mode perturbation. Structure is very evident at a 
wavelength of $120 \mu \mathrm{m}$, which is generated from the nonlinear interaction of $k_{1}-k_{2}$ modal components. Agreement between the experiment and the CALE simulation is very good, with the exception of the forward shock position, which is about $50 \mu \mathrm{m}$ ahead in the CALE simulation. Both the amplitude and general morphology of the interface structure are seen to be very well reproduced in the simulation. Future experiments of this type will be directed toward the continued evolution of the interface spectrum in order to more closely recreate the mixing conditions encountered in a SN explosion.

\section{CONCLUSION}

We have reported the results from a wide series of scaled laser experiments designed to test the validity of numerical codes used for the simulation of astrophysical phenomena such as supernova explosions. The need for such a validation stems from the rather long-standing discrepancy between observations and numerical simulation of SN 1987A. The experiments conducted thus far serve two purposes. They have helped to establish the range of phenomena relevant to the supernova mixing problem that can be addressed on a laser facility. Problems involving spherical divergence, multiple interfaces, three-dimensional and multi-mode interfaces could be studied in conventional gas-dynamic shock tubes, but not at the strong shock conditions of interest to astrophysics.

These experiments also serve as a first rough assessment of the validity of numerical codes used for the simulation of supernova hydrodynamics. To the level and resolution probed thus far, we find the result of numerical simulation to be in general agreement with the experiments. With improvement in the magnification and spatial resolution that have been recently demonstrated on laser experiments, ${ }^{60}$ we expect to be able to improve these results to the point that we can begin to differentiate between the results of different codes.

Having established a capability for performing relevant scaled experiments in the strong shock regime of interest, we can now begin to extend the range of problems and physics that can be addressed. The recent work of Khokhlov, for example, suggests that the supernova mixing problem may be far from spherical, with strong polar jets and Mach disks accelerating the inner layers to velocities high enough to explain the observations. Having established that relatively clean spherically divergent experiments may be performed, one can now begin to alter the experimental geometry to address the physics introduced by aspherical explosions. We hope to be able to use this testbed to continue to address new ideas as they are introduced in the astrophysical community and to provide benchmark data to augment observation, theory, and numerical modeling.

\section{ACKNOWLEDGMENTS}

Work performed under the auspices of the U.S. Department of Energy Lawrence Livermore National Laboratory under Contract No. W-7405-ENG48 and additional funding to the University of Michigan from the U.S. Department of Energy.

${ }^{1}$ T. Dotani, K. Hayashida, H. Inoue et al., Nature (London) 330, 230 (1987).

${ }^{2}$ R. Sunyaev, A. Kaniovsky, V. Efremov et al., Nature (London) 330, 227 (1987).

${ }^{3}$ M. Itoh, S. Kumagai, T. Shigeyama, K. Nomoto, and J. Nishimura, Nature (London) 330, 233 (1987).

${ }^{4}$ W. D. Arnett, J. N Bahcall, R. P. Kershner, and S. E. Woolsey, Annu. Rev. Astron. Astrophys. 27, 629 (1989).

${ }^{5}$ R. A. Chevalier, Nature (London) 355, 691 (1992).

${ }^{6}$ F. C. Witteborn, J. D. Bregman, D. H. Wooden, P. A. Pinto, D. M. Rank, S. E. Woosley, and M. Cohen, Astrophys. J. Lett. 338, L9 (1989).

${ }^{7}$ J. Tueller, S. Barthelmy, N. Gehrels, B. J. Teegarden, M. Leventhal, and C. J. MacCallum, Astrophys. J. Lett. 351, L41 (1990).

${ }^{8}$ I. Hachisu, T. Matsuda, K. Nomoto, and T. Shigeyama, Astrophys. J. Lett. 368, L27 (1991).

${ }^{9}$ B. A. Fryxell, E. Müller, and D. Arnett, Astrophys. J. 367, 619 (1991).

${ }^{10}$ E. Müller, B. A. Fryxell, and D. Arnett, Astron. Astrophys. 251, 505 (1991).

${ }^{11}$ M. Herant and W. Benz, Astrophys. J. Lett. 370, L81 (1991).

${ }^{12}$ M. Herant and W. Benz, Astrophys. J. 387, 294 (1992).

${ }^{13}$ R. A. Chevalier, Astrophys. J. 207, 872 (1976).

${ }^{14}$ Lord Rayleigh, Scientific Papers II (Cambridge, England, 1900), p. 200.

${ }^{15}$ G. I. Taylor, Proc. R. Soc. London, Ser. A 201, 192 (1950).

${ }^{16}$ R. D. Richtmyer, Commun. Pure Appl. Math. 13, 297 (1960).

${ }^{17}$ E. E. Meshkov, Izv. Acad. Sci. USSR Fluid Dynamics 4, 101 (1969).

${ }^{18}$ K. Kifonidis, T. Plewa, H.-Th. Janka, and E. Müller, Astrophys. J. Lett. 531, L123 (2000).

${ }^{19}$ G. Bazan and W. D. Arnett, Astrophys. J. Lett. 433, L41 (1994).

${ }^{20}$ G. Bazan and W. D. Arnett, Astrophys. J. 496, 316 (1998).

${ }^{21}$ W. D. Arnett, Ann. N.Y. Acad. Sci. 898, 77 (2000).

${ }^{22}$ J. P. Dahlburg, J. H. Gardner, G. D. Doolen, and S. W. Haan, Phys. Fluids B 5, 571 (1993).

${ }^{23}$ J. Hecht, U. Alon, and D. Shvarts, Phys. Fluids 6, 4019 (1994).

${ }^{24}$ M. M. Marinak, B. A. Remington, S. V. Weber, R. E. Tipton, S. W. Haan, K. S. Budil, O. L. Landen, J. D. Kilkenny, and R. Wallace, Phys. Rev. Lett. 75, 3677 (1995).

${ }^{25}$ T. Yabe, H. Hoshino, and T. Tsuchiya, Phys. Rev. A 44, 2756 (1991).

${ }^{26}$ J. Kane, D. Arnett, B. A. Remington, S. G. Glendinning, G. Bazan, E. Müller, B. A. Fryxell, and R. Teyssier, Astrophys. J. 528, 989 (2000).

${ }^{27}$ A. M. Khokhlov, P. F. Hoflich, E. S. Oran, J. C. Wheeler, and L. Wang, Astrophys. J. Lett. 524, L107 (1999).

${ }^{28}$ J. J. Quirk, Int. J. Numer. Methods Fluids 18, 555 (1994).

${ }^{29}$ R. L. Holmes, G. Dimonte, B. Fryxell, M. L. Gittings, J. W. Grove, M. Schneider, D. H. Sharp, A. L. Velikovich, R. P. Weaver, and Q. Zhang, J. Fluid Mech. 389, 55 (1999).

${ }^{30}$ J. Kane, D. Arnett, B. A. Remington, S. G. Glendinning, J. Castor, R. Wallace, A. Rubenchik, and B. A. Fryxell, Astrophys. J. Lett. 478, L75 (1997).

${ }^{31}$ B. A. Remington, J. Kane, R. P. Drake et al., Phys. Plasmas 4, 1994 (1997).

${ }^{32}$ R. P. Drake, J. Geophys. Res. 104, 14505 (1999).

${ }^{33}$ J. Kane, D. Arnett, B. A. Remington, S. G. Glendinning, G. Bazan, R. P. Drake, B. A. Fryxell, R. Teyssier, and K. Moore, Phys. Plasmas 6, 2065 (1999).

${ }^{34}$ B. A. Remington, R. P. Drake, D. Arnett, and H. Takabe, Science 284, 1488 (1999).

${ }^{35}$ B. A. Remington, R. P. Drake, D. Arnett, and H. Takabe, Phys. Plasmas 7, 1641 (2000).

${ }^{36}$ J. W. Connor and J. B. Taylor, Nucl. Fusion 17, 1067 (1977).

${ }^{37}$ D. D. Ryutov, R. P. Drake, J. Kane, E. Liang, B. A. Remington, and M. W. Wood-Vasey, Astrophys. J. 518, 821 (1999).

${ }^{38}$ D. D. Ryutov, B. A. Remington, H. F. Robey, and R. P. Drake, Phys. Plasmas 8, 1804 (2001).

${ }^{39}$ J. T. Larsen and S. M. Lane, J. Quant. Spectrosc. Radiat. Transf. 51, 179 (1994).

${ }^{40}$ T. R. Boehly, R. S. Craxton, T. H. Hinterman et al., Rev. Sci. Instrum. 66, 508 (1995).

${ }^{41}$ J. M. Soures, R. L. McCrory, C. P. Verdon et al., Phys. Plasmas 3, 2108 (1996). 
${ }^{42}$ T. R. Boehly, D. L. Brown, R. S. Craxton et al., Opt. Commun. 133, 495 (1997).

${ }^{43}$ D. K. Bradley, J. A. Delettrez, R. Epstein et al., Phys. Plasmas 5, 1870 (1998).

${ }^{44}$ K. S. Budil, T. S. Perry, P. M. Bell, J. D. Hares, P. L. Miller, T. A. Peyser, R. Wallace, H. Louis, and D. E. Smith, Rev. Sci. Instrum. 67, 485 (1996).

${ }^{45}$ R. T. Barton, The CALE computer code, in Numerical Astrophysics (Jones and Bartlett, Boston, 1985), p. 482.

${ }^{46}$ R. P. Drake, H. F. Robey, O. A. Hurricane, B. A. Remington, J. Knauer, D. Arnett, D. D. Ryutov, J. O. Kane, K. S. Budil, and J. Grove, Astrophys. J. (to be published).

${ }^{47}$ J. Kane, H. F. Robey, B. A. Remington et al., Phys. Rev. E (to be published).

${ }^{48}$ M. Brouillette and R. Bonazza, Phys. Fluids 11, 1127 (1999).

${ }^{49}$ N. C. Freeman, Proc. R. Soc. London, Ser. A 228, 341 (1955).

${ }^{50}$ J. J. Erpenbeck, Phys. Fluids 5, 1181 (1962).
${ }^{51}$ D. Ryu and E. T. Vishniac, Astrophys. J. 313, 820 (1987).

${ }^{52}$ M. Mac Low and M. L. Norman, Astrophys. J. 407, 207 (1993)

${ }^{53}$ J. Grun, J. Stamper, C. Manka, J. Resnick, R. Burris, J. Crawford, and B. H. Ripin, Phys. Rev. Lett. 66, 2738 (1991).

${ }^{54}$ R. A. Chevalier, Astrophys. J. 359, 463 (1990).

${ }^{55}$ Luo and R. A. Chevalier, Astrophys. J. 435, 815 (1994).

${ }^{56}$ R. Sari, E. Waxman, and C. Shvarts, Astrophys. J., Suppl. Ser. 127, 475 (2000).

${ }^{57}$ J. D. Lindl, Inertial Confinement Fusion (Springer-Verlag, New York, 1998), p. 54.

${ }^{58}$ J. Grun, R. Decoste, B. H. Ripin, and J. Gardner, Appl. Phys. Lett. 39, 545 (1981).

${ }^{59}$ B. Meyer and G. Thiell, Phys. Fluids 27, 302 (1984).

${ }^{60}$ O. Landen, D. R. Farley, S. G. Glendinning et al., Rev. Sci. Instrum. 72, 627 (2001). 